J. A. Fernley, R.F. Jameson, M.R. Sherrington University of Leicester, Leicester, United Kingdom

Abstract. Infrared photometry enables radii, and hence absolute magnitudes, to be derived more accurately than is possible using purely optical photometry. In this paper we present BVJHK observations of the galactic cepheids $T$ $\mathrm{Vu} 1, \mathrm{U}$ Vu 1 and $T$ Mon. Using this data we find reasonable agreement with current versions of the Period-Luminosity relations, in both the optical and infrared.

From these relations and existing optical and infrared data for LMC Cepheids we find for the LMC (i) $\mathrm{A}_{\mathrm{v}}=0.35$ and (ii) a distance modulus of 18.50. In addition we show that the $\mathrm{P}-\mathrm{L}-\mathrm{C}$ relation has the form

$\left\langle\mathrm{M}_{\mathrm{v}}>=-3.10 \log \mathrm{P}+1.70(\langle\mathrm{~B}\rangle-\langle\mathrm{V}\rangle)-2.37\right.$

1. Introduction

One of the methods of calibrating the Cepheid period-

luminosity relation is to use Baade-Wesselink techniques to obtain radii, and hence absolute magnitudes, for individual stars. Recently there have been several modifications to the original Baade-Wesselink technique (e.g., Balona 1977). In addition it has been shown by Fernley et al. (1984) that the accuracy with which radii can be derived using these techniques can be improved by using near infrared observations. We have obtained BVJHK photometry of the galactic Cepheids $T$ Vul and U VuI and using these data and Wisniewski and Johnson's(1968) BVJK photometry of the longer-period Cepheid $T$ Mon, we derive radii (section2) and absolute magnitudes (section 3). By plotting these results in Period-Luminosity diagrams, in both the optical and infrared, we derive the reddening and distance modulus to the LMC (section 4). Finally we use these results to derive the coefficients of the $\mathrm{P}-\mathrm{L}-\mathrm{C}$ relation (section 5 ).

2. Radii. To obtain radii we use the relation derived by Balona (1977)

$$
\Delta \mathrm{M}=\mathrm{a} \Delta \mathrm{C}+\mathrm{b} \Delta \mathrm{R}+\mathrm{c}
$$

where $\mathrm{a}, \mathrm{b}$ and $\mathrm{c}$ are constants, $\Delta \mathrm{M}$ the change in magnitude, $\Delta \mathrm{C}$ the change in a colour and $\Delta R$ the radius variation defined by 


$$
\Delta R=K P \int_{\phi_{1}}^{\phi_{2}}\left(V_{r}-\gamma\right) d \phi
$$

where $P$ is the period, $V$ the radial velocity deduced from the Doppler shifts of the spectral lines, $\gamma$ the centre of mass velocity of the star, $\phi$ the phase of the pulsation cycle and $K$ a conversion factor to account for geometrical projection and limb darkening. Following Karp (1975) we assign a value $\mathrm{K}=1.31$. The radial velocity data for the three stars was taken from Lust-Kulka (1954), Sanford $(1951,6)$ and Wallerstein (1972).

Using a maximum-likelihood technique we fit the observables $\Delta \mathrm{M}, \Delta \mathrm{C}$ and $\Delta \mathrm{R}$ in equation ( 1 ) to obtain values for the constants $\mathrm{a}, \mathrm{b}$ and $\mathrm{c}$. The constant $a$ is the slope of the colour-surface brightness relation and the constant $b$ is related to the mean radius, $\bar{R}$, of the star

$$
\mathrm{b}=\left(5 \log _{10} \mathrm{e}\right) / \overrightarrow{\mathrm{R}}
$$

Fernley et a1. (1984) showed that superior results could be obtained using infrared data. There are two reasons for this. Firstly infrared magnitudes are less temperature sensitive than optical magnitudes and are thus more sensitive to the radius variation. Secondly, the opticalinfrared colour indices ( $V-J, V-K$ ) have a larger amplitude than the purely optical indices $(B-V)$ and are thus more "stable" temperature indicators. There are illustrated in Figure 1.

\section{Absolute Magnitudes.}

We show our results in Table 1 . The radii have been discussed in the previous section. The reddenings are an average of various determinations as summarized by Feltz and McNamara (1980). The absolute magnitudes were obtained by two different methods. Firstly, by using the slopes of the colour-surface brightness relations, determined in the previous section, and the relation

$$
M=M_{\theta}+a\left[C-C_{\theta}\right]-5 \log R / R_{\theta}
$$

where $M$ and $C$ are the absolute magnitude and unreddened colour index respectively. A second method is to obtain $T_{\text {from }}(B-V)_{0}$, using the colour-temperature calibrations of Kraft $(1961)$ and Pe1 (1978), and then the relation

$$
M=M_{\theta}-2.5 \log \left[B(T) / B\left(T_{\theta}\right)\right]-5 \log R / R_{\theta}
$$

where $B(T)$ is the Planck function. This should be a good approximation at the infrared wavelengths. The two methods agree closely which suggests the slopes of the colour-surface brightness relation are reasonable. This is reassuring for the accuracy of the method of section 2 . 
Figure 1. The radius variation of $\mathrm{T}$ Vul derived from equation 2 (solid line) and from inverting equation 1 with the best-fit values of $a, b$ and $c(*=$ using $V$, $B-V$ and $x=$ using $K, V-K)$.

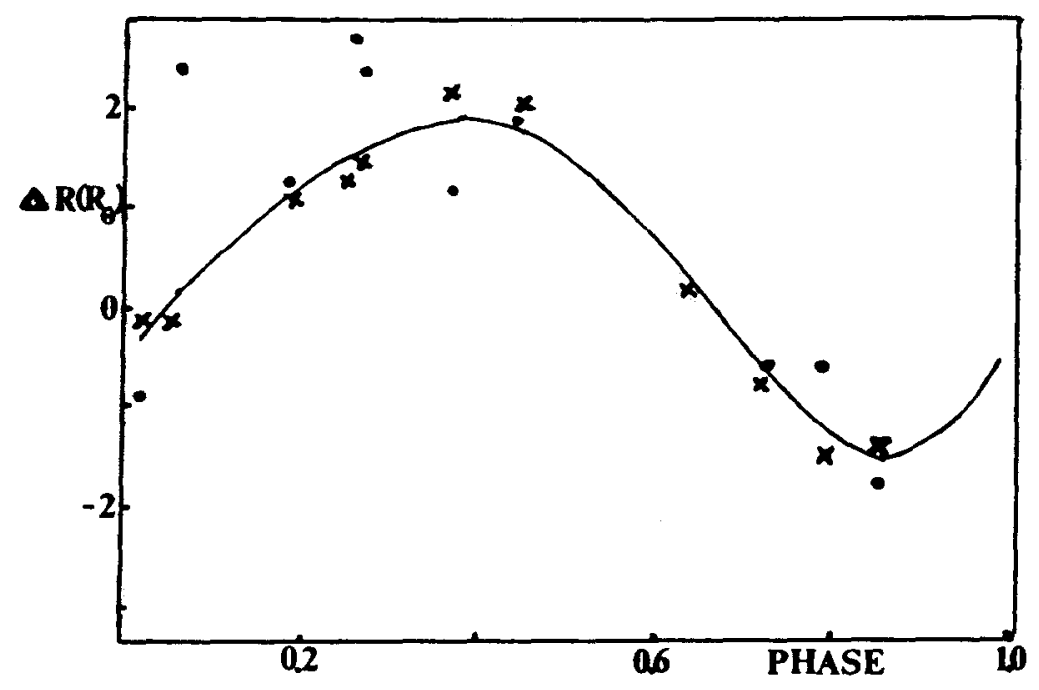

In Figure 2 we compare our results with two recent determinations of the Period-Luminosity relation. Stothers (1983) and McGonegal et al. (1983) used a cluster main-sequence fitting method to obtain their calibration. Their calibration is thus completely independent of ours and the good agreement between the two is encouraging. In the near future we hope to obtain photometry of several more Cepheids to test this agreement more fully.

\section{Table 1}
T Vul
U Vu1
T Mon

$\begin{array}{lccr}\mathrm{R}\left(\mathrm{R}_{\theta}\right) & 37.70 \pm 2.50 & 63.40 \pm 5.00 & 147.50 \pm 14.00 \\ (\mathrm{~B}-\mathrm{V})_{0} & 0.68 \pm 0.02 & 0.82 \pm 0.05 & 1.10 \pm 0.05 \\ \mathrm{E}(\mathrm{B}-\mathrm{V}) & 0.12 \pm 0.01 & 0.65 \pm 0.04 & 0.33 \pm 0.04 \\ \mathrm{~T}_{\mathrm{e}} & 5800 \pm 100 & 5440 \pm 150 & 4900 \pm 150 \\ \left\langle\mathrm{M}_{\mathrm{v}}\right\rangle & -3.33 \pm 0.23 & -4.06 \pm 0.29 & -5.54 \pm 0.35 \\ \left\langle\mathrm{M}_{\mathrm{H}}\right\rangle & -4.47 \pm 0.16 & -5.49 \pm 0.19 & -7.14 \pm 0.26 \\ \log \mathrm{P} & 0.646 & 0.902 & 1.432\end{array}$

Note: $\mathrm{R},(\mathrm{B}-\mathrm{V})_{0}$ and $\mathrm{T}_{\mathrm{e}}$ are at phase $\phi=0.7$ (minimum 1ight) 
Figure 2. Period-Luminosity relations for galactic Cepheids in the optical and infrared. ${ }^{*}=$ Stothers 1983 (V), McGonegal et al. 1983 (H), $\mathrm{x}=$ this work.

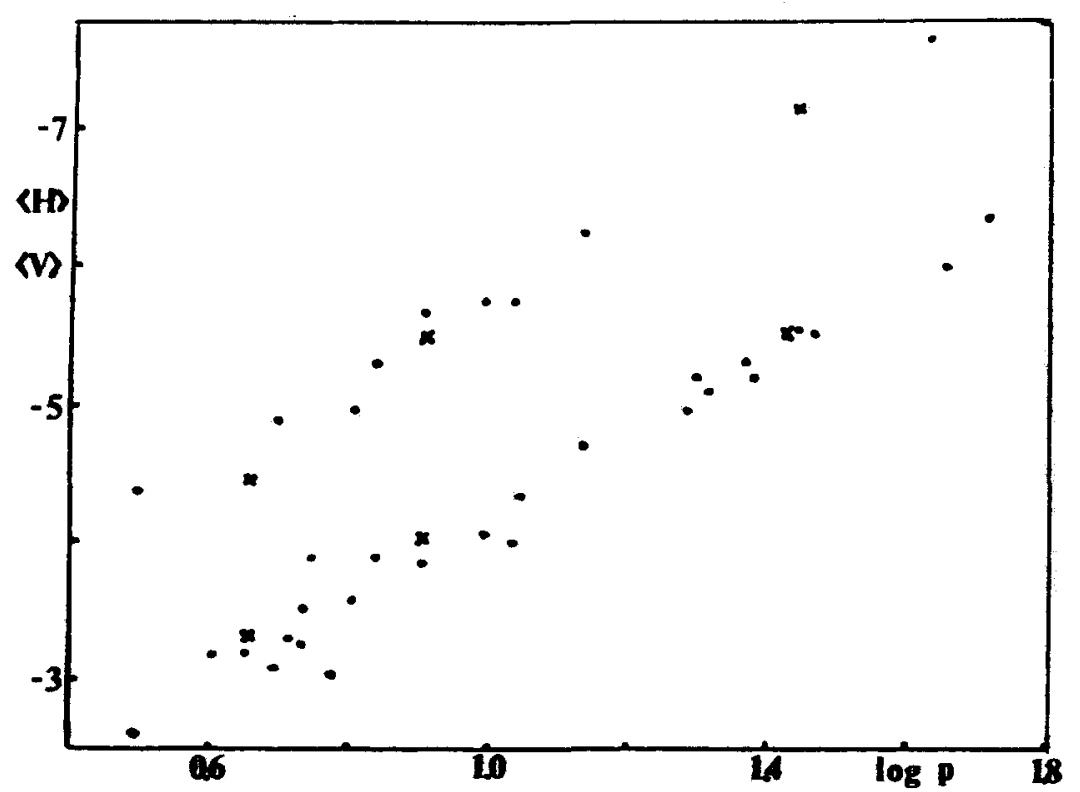

4. Distance to the LMC.

Fitting our results to the slopes of existing optical and infrared Period-Luminosity relations for LMC Cepheids (Mart in et al 1979, McGonegal et al 1982) we find distance moduli of $\mathrm{V}-\mathrm{M}_{\mathrm{v}}=18.85 \pm$ 0.15 and $h-H=18.55 \pm 0.14$. For $A A_{h}=7 \pm 1$ (Jones and Hyland 1980) and two distance moduli converge to $18.50 \pm 0.10$ for $A=0.35$. This higher value for the reddening confirms the estimate of de Vaucouleurs (1978), however, the distance modulus is approximately 0.2 larger than he derives from the average of various indicators.

\section{P-L-C Relation.}

A recent determination of the $\mathrm{P}-\mathrm{L}-\mathrm{C}$ relation is due to Martin et al. (1979) who apply a maximum-1ikelihood technique to the unreddened optical data on LMC Cepheids. They find

$$
\langle\mathrm{V}\rangle=-3.80 \log \mathrm{P}+2.70(\langle\mathrm{~B}\rangle-\langle\mathrm{V}\rangle)+16.41
$$

This is incompatible with the results of McGonegal et al.(1982) and ourselves. McGonegal et al. (1982) find for the LMC Cepheids

$$
<\mathrm{H}>=-3.10 \log \mathrm{P}+15.90
$$

We find, from Section 2, the slopes of the colour-surface brightness relation for $V, B-V$ and $H, B-V$. Thus we may write

$$
\begin{aligned}
& \langle V\rangle=-5 \log R / R_{\theta}+2.20(\langle B\rangle-\langle V\rangle)+3.40 \\
& \left\langle H>-5 \log / R_{O}+0.50(\langle B\rangle-\langle V\rangle)+3.17\right.
\end{aligned}
$$


where we have $\mathrm{V}_{\Theta}=4.83, \mathrm{H}_{\Theta}=3.48$ and $(\mathrm{B}-\mathrm{V})_{\theta}=0.65$. Combining (7) and (8) gives

$$
\langle\mathrm{V}\rangle=-3.10 \log \mathrm{P}+1.70(\langle\mathrm{~B}\rangle-\langle\mathrm{V}\rangle)+16.13
$$

The discrepancy between (6) and (9) is interpreted as partial confirmation of the arguments raised against the P-L-C (e.g., Stift 1982).

\section{References}

Balona, L.A., (1977), M.N.R.A.S., 178, 231.

Feltz, K.A., and McNamara, D.H., $(\overline{1980})$, P.A.S.P., 92, 609.

Fernley, J.A., Jameson, R.F., Sherrington, M.R., (1984), M.N.R.A.S. (in press)

Jones, T.J., and Hyland, A.R., (1980) M.N.R.A.S., 192, 359.

Karp, A.H., (1975), Ap.J., 201, 641.

Kraft, R.P., (1961), Ap.J., 134, 616.

Lust-Ku1ka, R., (1954), Z. Ast., 33, 211.

Martin, W.L., Warren, P.R., Feast, M.W., (1979), M.N.R.A.S., 188, 139.

McGonega1, R., McLaren, R.A., McAlary, C.W., Madore, B.F., (1 $\overline{982})$ Ap.J., 257, L33.

McGonega1, R., McLaren, R.A., McAlary, C.W., Madore, B.F., (1983), Ap.J., 269, 641 .

Pe1, J.W., (1978) Astr. Ap., 62, 75.

Sanford, R.F., (1951), Ap. J. 114, 331.

Sanford, R.F., (1956), Ap. J. 123, 201.

Stift, M.J., (1982), Astr. Ap. $\overline{112}, 149$.

Stothers , R.B., (1983), Ap. J., 274, 20.

de Vaucouleurs, G., (1978), Ap. J., 223, 730.

Wallerstein, G., (1972), P.A.S.P., 84, 656 .

Wisniewski, W.Z., and Johnson, H.L., (1968), Lunar and Planetary Lab. Comm., No. 112. 